\title{
In-vitro Studies of Artificially Removed Human Renal Stones in the Economic Age Group (30-40years)
}

\author{
P. Sundaramoorthi *, S. Kalainathan**, G. Kanchana***, R. Santhi * \\ *Department of Physics, Mahendra Engineering College, Mallasamudram (W), Namakkal, India. \\ (E-mail: sundara78@rediffmail.com) \\ **Department of Physics, Vellore Institute of Technology, Vellore, India \\ ***Department of Bio-Chemistry, MCAS, Rasipuram, Namakkal, India.
}

\begin{abstract}
Electrical and thermal conductivity studies play a vital role in the field of science and technology. According to the conductivity terms, materials can be classified in to good conductors, bad conductors, semiconductors and super conductors. In the free electron theory, electron only acts as carrier and carries its energy from one point to another point either natural or forced. In the present investigation, five renal stones are collected from the poor hard working males who are affected with mineral deposition in the urinary tracts. The stones are collected from the hospital by Lithotropic treatment process. Using two-probes method, the electrical conductivities are measured at different temperatures. The thermal conductivity and temperature coefficients are calculated. The results are reported and discussed.
\end{abstract}

\section{Introduction}

The conductivity of many biomaterials has been reported [1]. The applied problems, such as thrombogenesis and enzymatic activity of cytochrome oxides, have been dealt with either semi conductivity or electrets behavior of different biomaterials. In the present study, both these aspect have been attempted on the same materials, namely renal stones or renal calculi. The study of electrets behaviors and conductivity becomes essential in order to find an inhibitor for renal stones or prevent its growth. The electrets behavioral of the renal stone material was studied through TSD, TSP $[2,3,4]$. Here the investigators report the D.C conductivity of kidney stones as a function of temperature and applied electric field and its interpretations. 


\section{Materials and Methods}

Kidney stones are removed from the affected patients by Litho tropic process in the Rasi stones diagnosing center in Rasipuram, Namakkal, India, and are used in the present investigations. These stones constituents are analyzed by biochemical analysis process. The stone constituents are reported in the Table 1. The major constituents of the samples are calcium phosphate and / or oxalate ions bond to an organic and sulponated muscoproteins [5]. The investigation results on TSP, TSD are reproducible from sample to sample of stone. However it varied slightly from stone to stone of either oxalate contents or phosphate contents depending upon the stone compositions. Due to the lengthy process of conductivity measurement, only five samples were used (Figure 1 through 5).

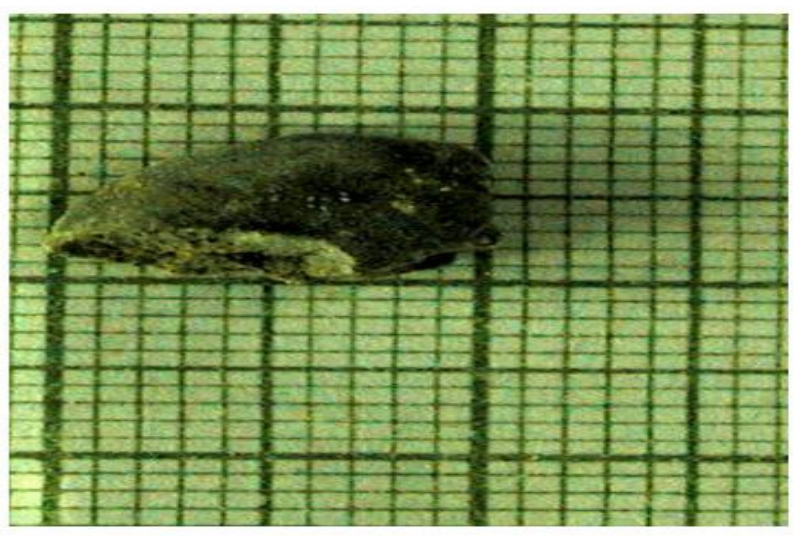

Stone-1 (10mmx8mmx3mm)

Figure 1. Sample A renal stone. 


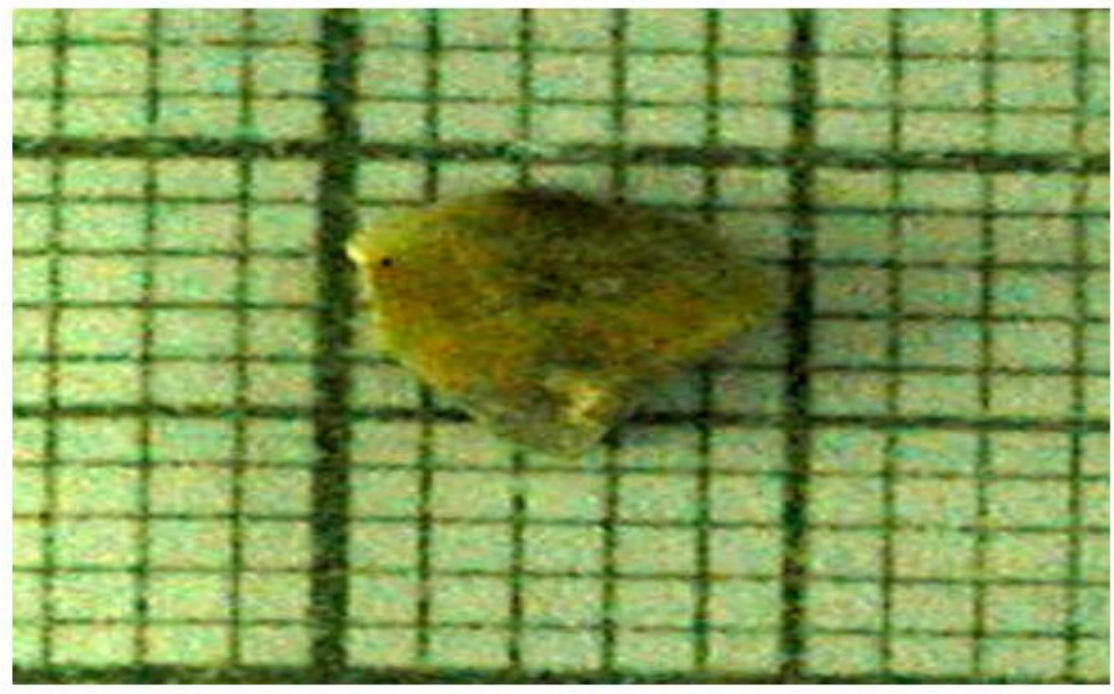

Stone-2 (9mmx6mmx10mm)

Figure 2. Sample B renal stone.

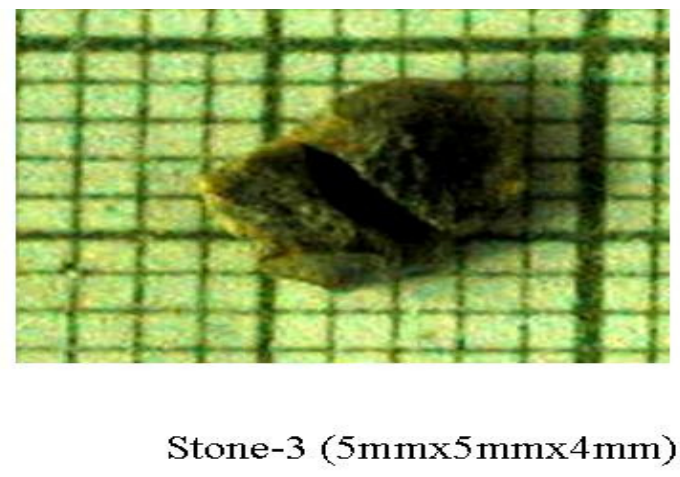

Figure 3. Sample C renal stone. 


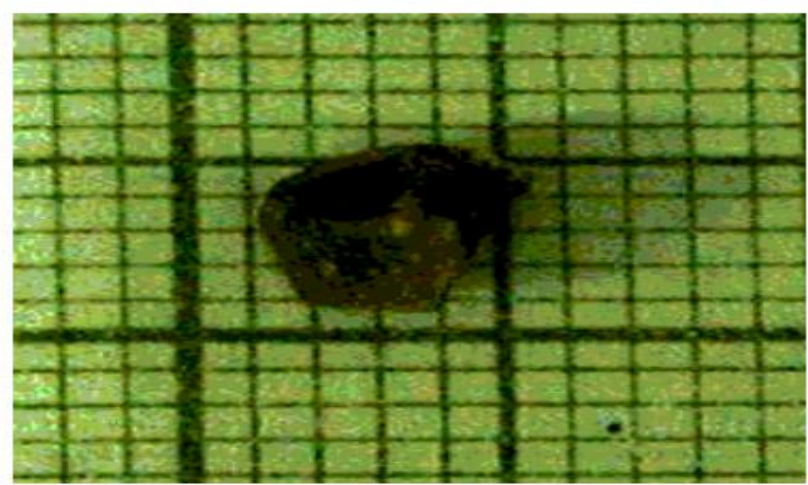

Stone-4 (4mmx5mmx4mm)

Figure 4. Sample D renal stone.

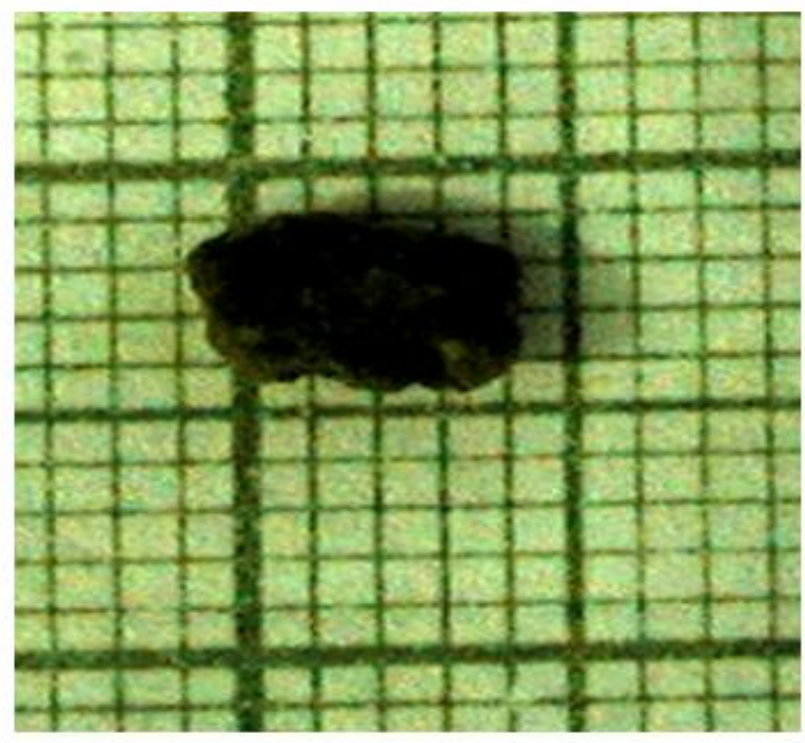

Stone-5 (5mmx4mmx3mm)

Figure 5. Sample E renal stone. 
Table 1. Chemical composition present in the renal stones

\begin{tabular}{|c|l|}
\hline Stones & Chemical constituents \\
\hline A & Calcium oxalate di-hydrate, calcium phosphate. \\
\hline B & Calcium oxalate monohydrate, Calcium oxalate di-hydrate. \\
\hline C & Calcium oxalate monohydrate Phosphates. \\
\hline D & Calcium oxalate monohydrate, Phosphate, Calcium oxalate di-hydrate. \\
\hline E & Calcium oxalate monohydrate \\
\hline
\end{tabular}

Stone samples are prepared for conductivity measurement initially. The bloodstains and other impurities are removed from the surfaces of the sample and dried naturally. Then the sample surfaces are smoothened by using very thin grain grinder. The renal stone samples are placed in between two aluminum probes with deep contact. The sample holders with the sample are kept inside the micro oven, which is automatically heated with electronic temperature monitor and controller (the accuracy is $2^{\circ} \mathrm{C}$ ). The D.C conductivity are studied at the temperature (T) from 295 $\mathrm{K}$ to $373 \mathrm{~K}$ at a irregular temperature intervals. The electric field applied to the probes starts from $1 \mathrm{~V}$ to $20 \mathrm{Vand}$ the corresponding currents are measured. All the sample are kept at a particular temperature at least 15 minutes to reach the saturation. Then the measurements are started. At higher voltage and higher temperature the current settling time are observed. The transient current of the sample mainly depends upon the presence of carriers in a sample according to the CurieVon-Schweidlar law. The conductivity has been reported for polymer samples [6-8] and ionic materials [9-10].

\section{Results}

The approximate area of each samples are measured by using graph sheet.

The temperatures are varied from $295 \mathrm{~K}$ to $373 \mathrm{~K}$. The applied voltage (V), corresponding current measurements (I), resistance $(R)$, resistivity $(\rho)$, conductivity $(\sigma)$ and current density $(J)$ of each sample at a particular temperature are reported in Tables 2 through 16. 
Table 2. Electrets properties of renal stone at $30^{\circ} \mathrm{C}, A=152 \times 10^{-6} \mathrm{~m}^{2}, \mathrm{~L}=10 \times 10^{-3} \mathrm{~m}$

\begin{tabular}{|c|c|c|r|l|l|}
\hline S.NO & $\begin{array}{l}\text { Voltage applied } \\
\text { in Volts (V). }\end{array}$ & $\begin{array}{l}\text { Current measured in } \\
\text { micro -amperes (I). }\end{array}$ & $\begin{array}{l}\text { Resistance } \\
10^{+6} \\
(\mathrm{Ohm}) .\end{array}$ & $\begin{array}{l}\text { Resistivity in } \\
10^{7} \text { Ohm. M } \\
(\rho) .\end{array}$ & $\begin{array}{l}\text { Current density } \\
(\mathrm{J}) . \\
\mathrm{Amp} / \mathrm{m}^{2}\end{array}$ \\
\hline & & 4 & 1 & 6.5789 & 0.026 \\
1. & 4 & 4 & 1.25 & 8.2236 & 0.026 \\
2. & 5 & 5 & 1.2 & 7.8947 & 0.033 \\
3. & 6 & 6.5 & 1.076 & 7.0789 & 0.039 \\
4. & 7 & 7 & 1.142 & 7.5131 & 0.046 \\
5. & 8 & 8 & 1.125 & 7.4013 & 0.053 \\
6. & 9 & 8 & 1.25 & 8.2236 & 0.053 \\
7. & 10 & & & \\
\hline
\end{tabular}

$\mathrm{R}=1.149 \times 10^{6}$

$\rho=7.5591 \times 10^{7}$

$\mathrm{J}=0.0409$

Coefficient of electrical conductivity $(\sigma)=1.328 \times 10^{-8}$ mho.m-1

Table 3. Electrets properties of renal stone at $60^{\circ} \mathrm{C}, A=152 \times 10^{-6} \mathrm{~m}^{2}, \mathrm{~L}=10 \times 10^{-3} \mathrm{~m}$

\begin{tabular}{|c|c|c|c|c|c|}
\hline S.NO & $\begin{array}{l}\text { Voltage applied } \\
\text { in Volts (V). }\end{array}$ & $\begin{array}{l}\text { Current measured in } \\
\text { micro -amperes (I). }\end{array}$ & $\begin{array}{c}\text { Resistance } \\
10^{+6} \\
(\mathrm{Ohm}) .\end{array}$ & $\begin{array}{l}\text { Resistivity in } \\
10^{7} \text { Ohm. M } \\
(\rho) .\end{array}$ & $\begin{array}{l}\text { Current density } \\
(\mathrm{J}) . \text { Amp/m }\end{array}$ \\
\hline & & 3 & 1.33 & 8.767 & 0.021 \\
2. & 4 & 3 & 1.66 & 10.965 & 0.021 \\
3. & 5 & 4 & 1.5 & 9.8684 & 0.026 \\
4. & 6 & 5 & 1.4 & 9.2105 & 0.032 \\
5. & 7 & 6 & 1.333 & 8.7697 & 0.039 \\
6. & 8 & 7 & 1.285 & 8.4539 & 0.046 \\
7. & 10 & 7 & 1.428 & 9.3947 & 0.046 \\
\hline
\end{tabular}

$$
\mathrm{R}=1.4207 \times 10^{6} \quad \rho=9.3469 \times 10^{7} \quad \mathrm{~J}=0.033
$$

Coefficient of electrical conductivity $(\sigma)=1.0698 \times 10^{-8} \quad$ mho.m-1 
Table 4. Electrets properties of renal stone at $96^{\circ} \mathrm{C}, \mathrm{A}=152 \times 10^{-6} \mathrm{~m}^{2}, \mathrm{~L}=10 \times 10^{-3} \mathrm{~m}$

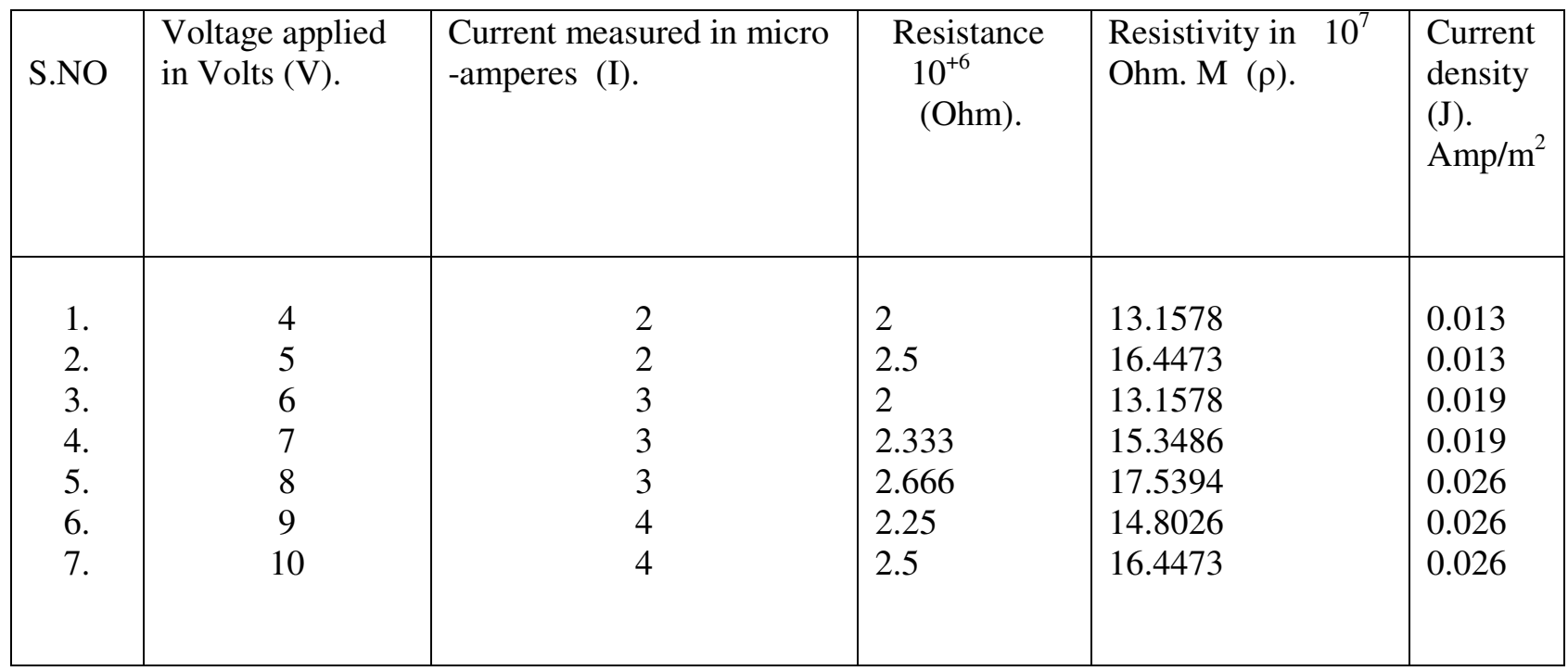

$$
\mathrm{R}=1.149 \times 10^{6} \quad \rho=15.2715 \times 10^{7} \quad \mathrm{~J}=0.0202
$$

Coefficient of electrical conductivity $(\sigma)=6.548 \times 10^{-9} \quad$ mho.m- 1

Table 5. Electrets properties of renal stone at $28^{\circ} \mathrm{C}, \mathrm{A}=33 \times 10^{-6} \mathrm{~m}^{2}, \mathrm{~L}=6 \times 10^{-3} \mathrm{~m}$

\begin{tabular}{|c|c|c|c|c|c|}
\hline S.NO & $\begin{array}{l}\text { Voltage applied } \\
\text { in Volts (V). }\end{array}$ & $\begin{array}{l}\text { Current measured in micro } \\
\text {-amperes (I). }\end{array}$ & $\begin{array}{c}\text { Resistance } \\
10^{+6} \\
(\mathrm{Ohm}) .\end{array}$ & $\begin{array}{l}\text { Resistivity in } 10^{7} \\
\text { Ohm. M }(\rho) .\end{array}$ & $\begin{array}{l}\text { Current } \\
\text { density } \\
(\mathrm{J}) \text {. } \\
\text { Amp } / \mathrm{m}^{2}\end{array}$ \\
\hline 1. & 3 & 100 & 0.03 & 5.4545 & 3.03 \\
\hline 2. & 4 & 110 & 0.0364 & 6.4182 & 3.33 \\
\hline 3. & 5 & 120 & 0.0417 & 7.5818 & 3.63 \\
\hline 4. & 6 & 140 & 0.0429 & 7.8000 & 4.24 \\
\hline 5. & 7 & 150 & 0.0467 & 8.4909 & 4.54 \\
\hline 6. & 8 & 165 & 0.0485 & 8.8182 & 5.10 \\
\hline 7. & 9 & 175 & 0.0514 & 9.3454 & 5.30 \\
\hline 8. & 10 & 180 & 0.0556 & 10.1090 & 5.45 \\
\hline
\end{tabular}

Coefficient of electrical conductivity $(\sigma)=1.2457 \times 10^{-7} \quad$ mho.m-1 
Table 6. Electrets properties of renal stone at $65^{\circ} \mathrm{C}, \mathrm{A}=33 \times 10^{-6} \mathrm{~m}^{2}, \mathrm{~L}=6 \times 10^{-3} \mathrm{~m}$

\begin{tabular}{|c|c|c|r|r|l|}
\hline S.NO & $\begin{array}{l}\text { Voltage applied } \\
\text { in Volts (V). }\end{array}$ & $\begin{array}{l}\text { Current measured in micro } \\
\text {-amperes (I). }\end{array}$ & $\begin{array}{l}\text { Resistance } \\
10^{+6} \\
(\text { Ohm }) .\end{array}$ & $\begin{array}{l}\text { Resistivity in } 10^{6} \\
\text { Ohm. M }(\rho) .\end{array}$ & $\begin{array}{l}\text { Current } \\
\text { density } \\
(\mathrm{J}) . \\
\text { Amp } / \mathrm{m}^{2}\end{array}$ \\
\hline 1. & 2 & 40 & 0.05 & 9.0909 & 1.12 \\
2. & 3 & 70 & 0.0429 & 7,8600 & 2.12 \\
3. & 4 & 80 & 0.05 & 9.0909 & 2.42 \\
4. & 5 & 100 & 0.0589 & 10.7690 & 2.57 \\
5. & 6 & 118 & 0.06 & 10.9090 & 3.03 \\
6. & 7 & 130 & 0.0659 & 10.7272 & 3.57 \\
7. & 8 & 140 & 0.0642 & 11.1818 & 3.93 \\
8. & 9 & 150 & 0.0667 & 11.6727 & 4.42 \\
9. & 10 & & & 12.1272 & 4.45 \\
\hline
\end{tabular}

Coefficient of electrical conductivity $(\sigma)=9.645 \times 10^{-8} \quad$ mho.m-1

$$
\mathrm{R}=0.05702 \times 10^{6} \quad \rho=10.3676 \times 10^{6} \quad \mathrm{~J}=3.07
$$

Table 7. Electrets properties of renal stone at $98^{\circ} \mathrm{C}, \mathrm{A}=33 \times 10^{-6} \mathrm{~m}^{2}, \mathrm{~L}=6 \times 10^{-3} \mathrm{~m}$

\begin{tabular}{|c|c|c|c|c|c|}
\hline S.NO & $\begin{array}{l}\text { Voltage applied } \\
\text { in Volts (V). }\end{array}$ & $\begin{array}{l}\text { Current measured in micro } \\
\text {-amperes (I). }\end{array}$ & $\begin{array}{l}\text { Resistance } \\
10^{+6} \\
(\mathrm{Ohm})\end{array}$ & $\begin{array}{l}\text { Resistivity in } 10^{6} \\
\text { Ohm. M }(\rho) .\end{array}$ & $\begin{array}{l}\text { Current } \\
\text { density } \\
(\mathrm{J}) . \\
\text { Amp } / \mathrm{m}^{2}\end{array}$ \\
\hline 1. & 2 & 25 & 0.1 & 1.8182 & 0.75 \\
\hline 2. & 3 & 35 & 0.0857 & 1.5582 & 1.06 \\
\hline 3. & 4 & 50 & 0.08 & 1.4545 & 1.51 \\
\hline 4. & 5 & 60 & 0.0833 & 1.5145 & 1.18 \\
\hline 5. & 6 & 65 & 0.0923 & 1.6782 & 1.96 \\
\hline 6. & 7 & 75 & 0.0933 & 1.6964 & 2.27 \\
\hline 7. & 8 & 90 & 0.0889 & 1.6164 & 2.73 \\
\hline 8. & 9 & 100 & 0.09 & 1.6364 & 3.63 \\
\hline 9. & 10 & 120 & 0.0833 & 1.5145 & 3.64 \\
\hline
\end{tabular}

Coefficient of electrical conductivity $(\sigma)=6.212 \times 10^{-8}$ mho.m-1 
Table 8. Electrets properties of renal stone at $30^{\circ} \mathrm{C}, A=22.5 \times 10^{-6} \mathrm{~m}^{2}, \mathrm{~L}=5 \times 10^{-3} \mathrm{~m}$

\begin{tabular}{|c|c|c|c|c|c|}
\hline S.NO & $\begin{array}{l}\text { Voltage applied } \\
\text { in Volts (V). }\end{array}$ & $\begin{array}{l}\text { Current measured in micro } \\
\text {-amperes (I). }\end{array}$ & $\begin{array}{c}\text { Resistance } \\
10^{+6} \\
(\mathrm{Ohm}) .\end{array}$ & $\begin{array}{l}\text { Resistivity in } 10^{6} \\
\text { Ohm. M }(\rho) .\end{array}$ & $\begin{array}{l}\text { Current } \\
\text { density } \\
(\mathrm{J}) \text {. } \\
\mathrm{Amp} / \mathrm{m}^{2}\end{array}$ \\
\hline 1. & 2 & 5 & 0.4 & 8.889 & 0.221 \\
\hline 2. & 5 & 7 & 0.571 & 12.6889 & 0.311 \\
\hline 3. & 6 & 10 & 0.6 & 13.333 & 0.444 \\
\hline 4. & 8 & 12 & 0.667 & 14.8222 & 0.533 \\
\hline 5. & 10 & 15 & 0.667 & 14.8222 & 0.666 \\
\hline 6. & 12 & 17 & 0.706 & 15.6889 & 0.755 \\
\hline 7. & 14 & 20 & 0.7 & 15.5516 & 0.889 \\
\hline 8. & 16 & 22 & 0.727 & 16.1556 & 0.978 \\
\hline 9. & 20 & 25 & 0.8 & 17.7778 & 1.111 \\
\hline
\end{tabular}

Coefficient of electrical conductivity $(\sigma)=6.937 \times 10^{-9} \quad$ mho.m- 1

Table 9. Electrets properties of renal stone at $58^{\circ} \mathrm{C}, \mathrm{A}=33 \times 10^{-6} \mathrm{~m}^{2}, \mathrm{~L}=6 \times 10^{-3} \mathrm{~m}$

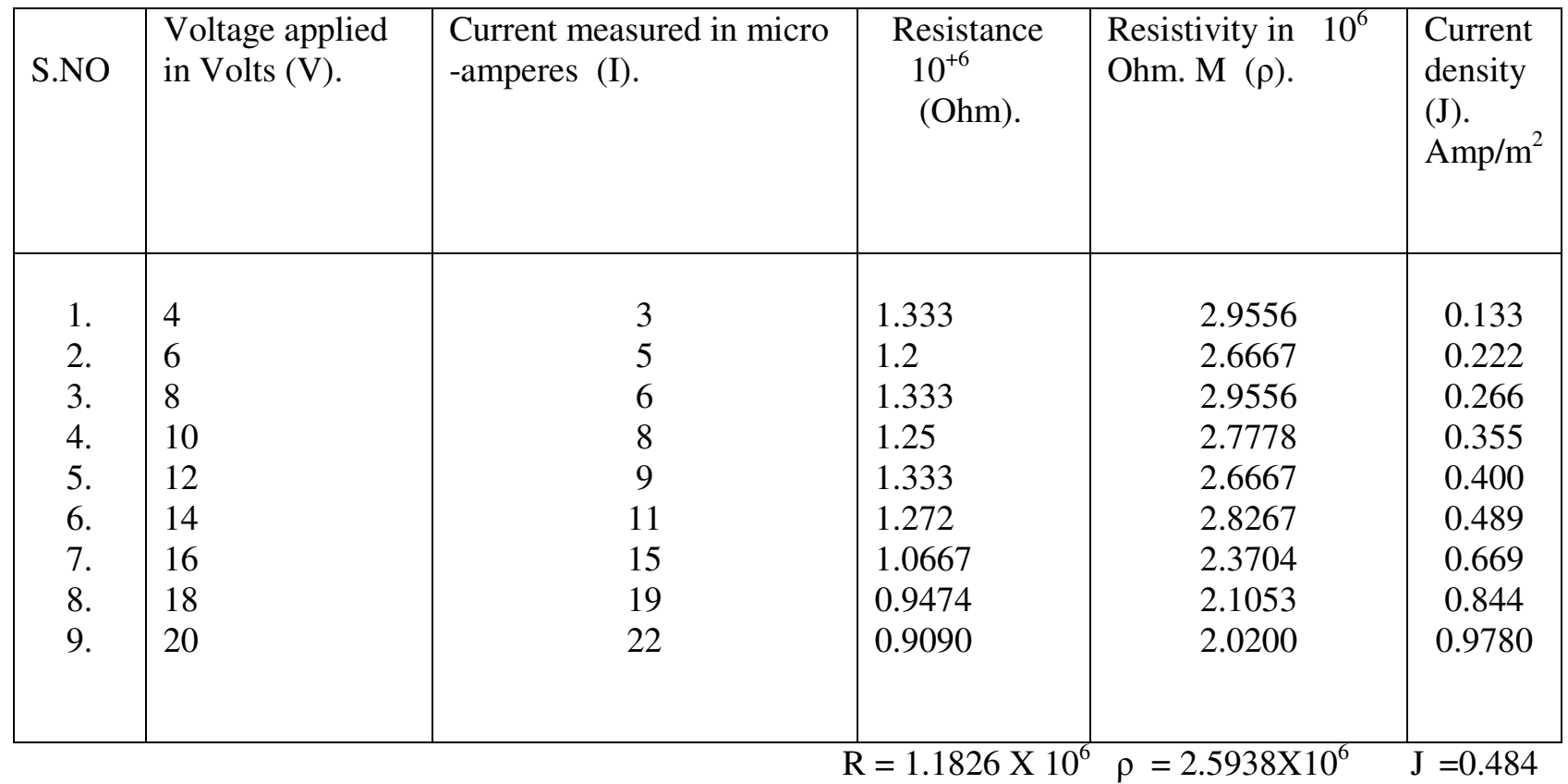

Coefficient of electrical conductivity $(\sigma)=3.855 \times 10^{-9} \quad$ mho.m-1 
Table 10. Electrets properties of renal stone at $95^{\circ} \mathrm{C}, \mathrm{A}=22.5 \times 10^{-6} \mathrm{~m}^{2}, \mathrm{~L}=5 \times 10^{-3} \mathrm{~m}$

\begin{tabular}{|c|c|c|c|c|c|}
\hline S.NO & $\begin{array}{l}\text { Voltage applied } \\
\text { in Volts (V). }\end{array}$ & $\begin{array}{l}\text { Current measured in micro } \\
\text {-amperes (I). }\end{array}$ & $\begin{array}{c}\text { Resistance } \\
10^{+6} \\
(\mathrm{Ohm})\end{array}$ & $\begin{array}{l}\text { Resistivity in } 10^{8} \\
\text { Ohm. M }(\rho) .\end{array}$ & $\begin{array}{l}\text { Current } \\
\text { density } \\
(\mathrm{J}) \text {. } \\
\text { Amp } / \mathrm{m}^{2}\end{array}$ \\
\hline $\begin{array}{l}1 . \\
2 . \\
3 . \\
4 . \\
5 . \\
6 . \\
7 . \\
8 . \\
9 .\end{array}$ & $\begin{array}{c}4 \\
6 \\
8 \\
10 \\
12 \\
14 \\
16 \\
18 \\
20\end{array}$ & $\begin{array}{c}2 \\
3 \\
4 \\
6 \\
7 \\
9 \\
13 \\
15 \\
18\end{array}$ & $\begin{array}{l}2 \\
3 \\
2 \\
1.667 \\
1.7142 \\
1.5556 \\
1.2308 \\
1.2 \\
1.111\end{array}$ & $\begin{array}{c}4.444 \\
6.6667 \\
4.4444 \\
3.7044 \\
3.8093 \\
3.4569 \\
2.6667 \\
2.4689 \\
2.1268\end{array}$ & $\begin{array}{c}0.88 \\
0.133 \\
0.177 \\
0.267 \\
0.311 \\
0.400 \\
0.578 \\
0.667 \\
0.800\end{array}$ \\
\hline
\end{tabular}

$$
\mathrm{R}=1.7198 \times 10^{6} \quad \rho=3.9577 \times 10^{6} \quad \mathrm{~J}=0.464
$$

Coefficient of electrical conductivity $(\sigma)=2.661 \times 10^{-9}$ mho.m- ${ }^{1}$

Table 11. Electrets properties of renal stone at $28^{\circ} \mathrm{C}, \mathrm{A}=20 \times 10^{-6} \mathrm{~m}^{2}, \mathrm{~L}=4 \times 10^{-3} \mathrm{~m}$

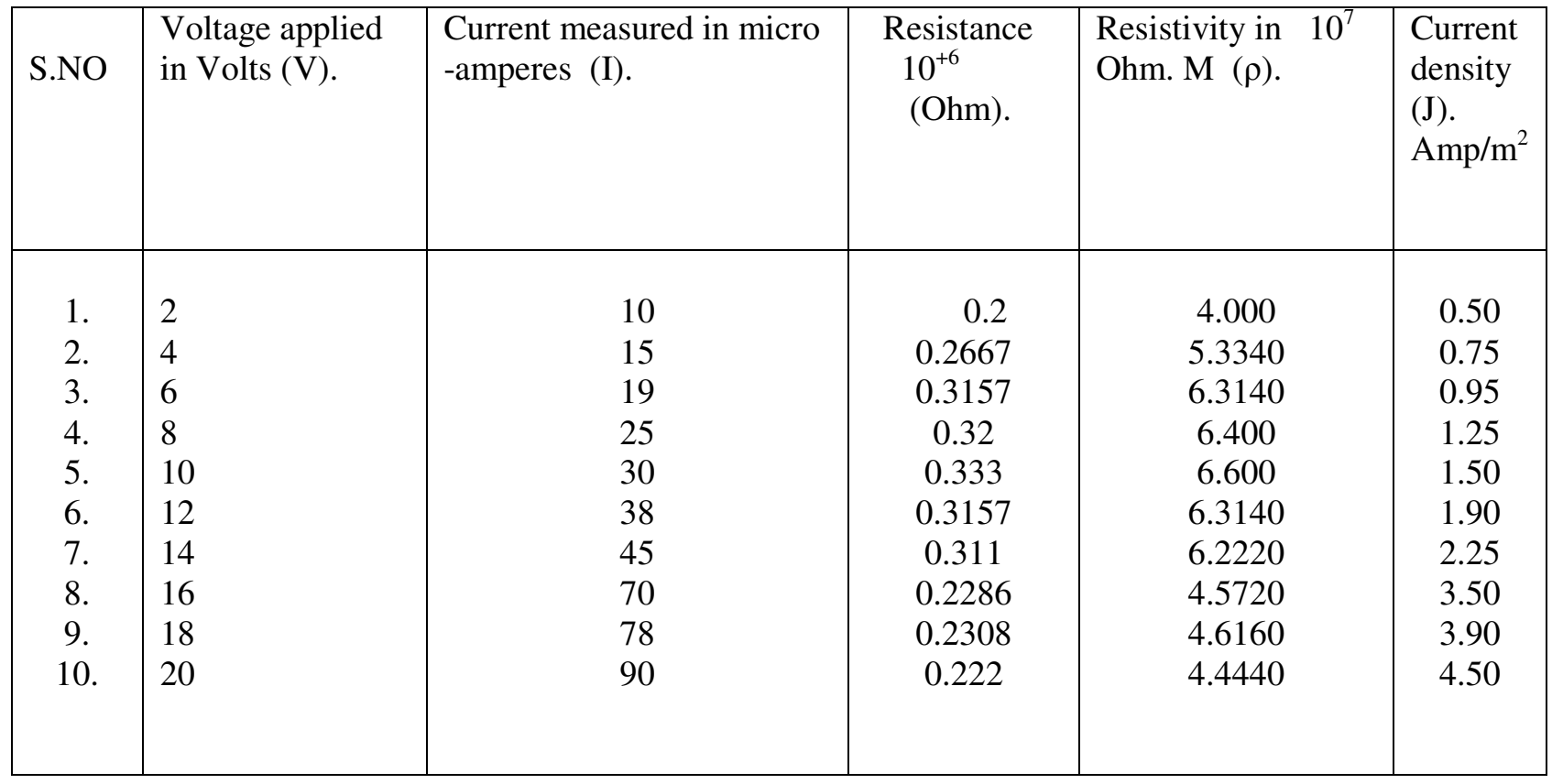

$$
R=0.2743 \times 10^{6} \quad \rho=5.4874 \times 10^{7} \quad J=2.049
$$

Coefficient of electrical conductivity $(\sigma)=1.822 \times 10^{-8}$ mho.m- ${ }^{1}$ 
Table 12. Electrets properties of renal stone at $70^{\circ} \mathrm{C}, \mathrm{A}=20 \times 10^{-6} \mathrm{~m}^{2}, \mathrm{~L}=4 \times 10^{-3} \mathrm{~m}$

\begin{tabular}{|c|l|c|c|c|c|}
\hline S.NO & $\begin{array}{l}\text { Voltage applied } \\
\text { in Volts (V). }\end{array}$ & $\begin{array}{l}\text { Current measured in micro } \\
\text {-amperes (I). }\end{array}$ & $\begin{array}{c}\text { Resistance } \\
10^{+6} \\
(\mathrm{Ohm}) .\end{array}$ & $\begin{array}{l}\text { Resistivity in } \\
\text { Ohm. M }(\rho) .\end{array}$ & $\begin{array}{l}\text { Current } \\
\text { density } \\
(\mathrm{J}) . \\
\text { Amp } / \mathrm{m}^{2}\end{array}$ \\
\hline & & & & & \\
1. & 2 & 6 & 0.333 & 6.6600 & 0.30 \\
2. & 4 & 10 & 0.4 & 8.000 & 0.50 \\
3. & 6 & 15 & 0.4 & 8.000 & 1.10 \\
4. & 8 & 20 & 0.4 & 8.000 & 1.30 \\
5. & 10 & 26 & 0.3847 & 7.6940 & 1.60 \\
6. & 12 & 32 & 0.375 & 7.5000 & 2.00 \\
7. & 14 & 40 & 0.35 & 7.000 & 2.60 \\
8. & 16 & 52 & 0.3077 & 6.1540 & 2.70 \\
9. & 18 & 60 & 0.3 & 6.000 & 3.00 \\
10. & 20 & 75 & 0.2667 & 5,3340 & 3.75 \\
& & & & & \\
\hline
\end{tabular}

Coefficient of electrical conductivity $(\sigma)=1.422 \times 10^{-8}$ mho.m-1

$$
\mathrm{R}=0.3517 \times 10^{6} \quad \rho=7.0342 \times 10^{7} \quad \mathrm{~J}=1.885
$$

Table 13. Electrets properties of renal stone at $98^{\circ} \mathrm{C}, \mathrm{A}=20 \times 10^{-6} \mathrm{~m}^{2}, \mathrm{~L}=4 \times 10^{-3} \mathrm{~m}$

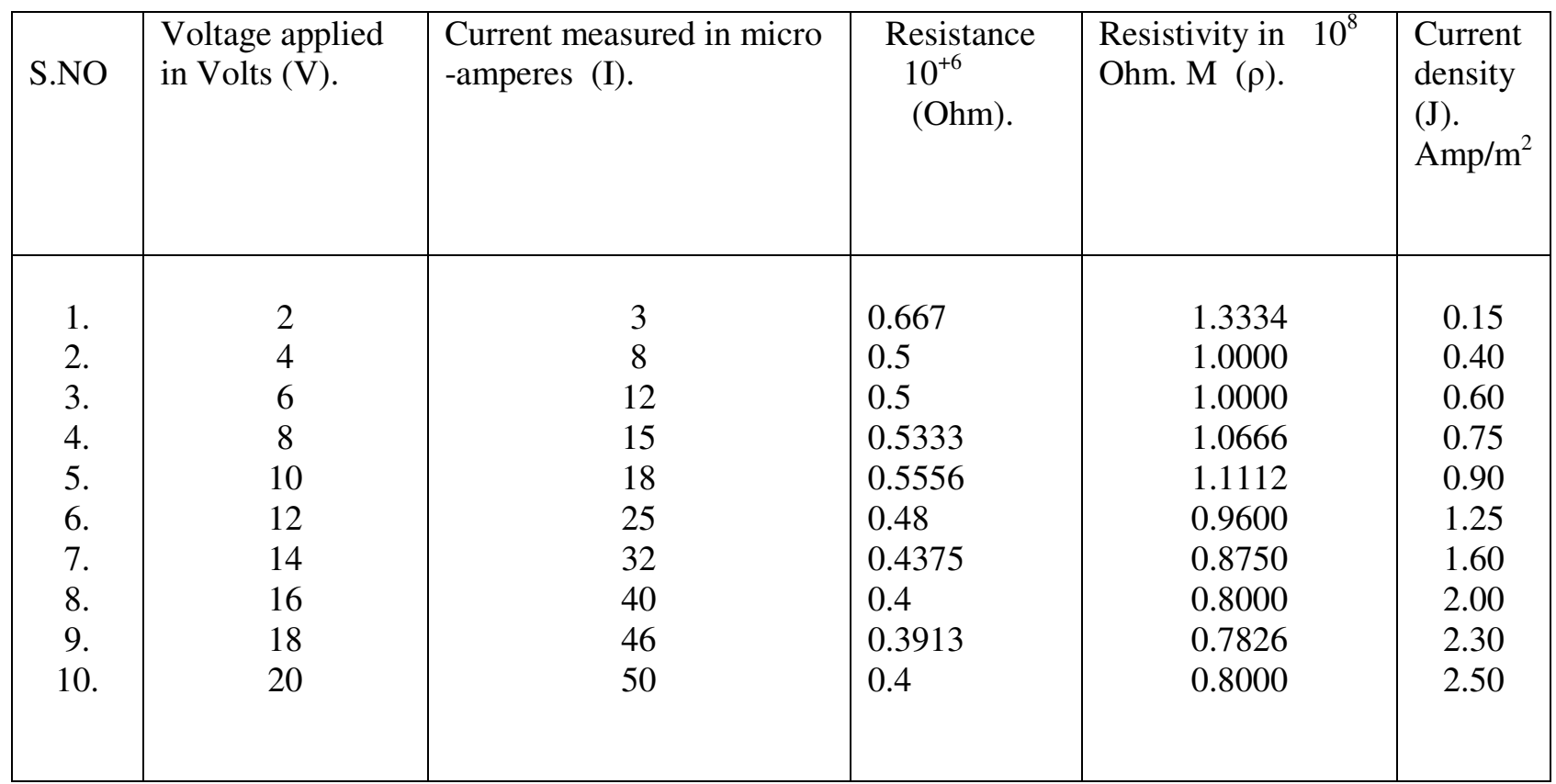

Coefficient of electrical conductivity $(\sigma)=1.0279 \times 10^{-9}$ mho.m-1

$R=0.4864 X 10^{6} \quad \rho=0.9728 \times 10^{8} \quad J=1.246$ 
Table 14. Electrets properties of renal stone at $28^{0} \mathrm{C}, A=9 \times 10^{-6} \mathrm{~m}^{2}, \mathrm{~L}=3 \times 10^{-3} \mathrm{~m}$

\begin{tabular}{|c|l|c|l|c|c|}
\hline S.NO & $\begin{array}{l}\text { Voltage applied } \\
\text { in Volts (V). }\end{array}$ & $\begin{array}{l}\text { Current measured in micro } \\
\text {-amperes (I). }\end{array}$ & $\begin{array}{l}\text { Resistance } \\
10^{+6} \\
(\mathrm{Ohm}) .\end{array}$ & $\begin{array}{l}\text { Resistivity in } \\
\text { Ohm. M }(\rho) .\end{array}$ & $\begin{array}{l}\text { Current } \\
\text { density } \\
(\mathrm{J}) . \\
\text { Amp } / \mathrm{m}^{2}\end{array}$ \\
\hline & & & & & \\
1. & 2 & 26 & 0.0769 & 2.5633 & 3.32 \\
2. & 4 & 35 & 0.1142 & 3.5067 & 3.88 \\
3. & 6 & 45 & 0.125 & 4.1667 & 5.33 \\
4. & 8 & 0.1230 & 4.1000 & 5,00 \\
5. & 10 & 78 & 0.1282 & 4.2733 & 8.66 \\
6. & 12 & 0.133 & 4.4433 & 10.00 \\
7. & 14 & 90 & 0.31284 & 5.2800 & 12.11 \\
8. & 16 & 109 & 0.1311 & 4.3700 & 13.55 \\
9. & 18 & 122 & 0.1286 & 4.2867 & 15.55 \\
10. & 20 & 140 & 0.1176 & 3.9200 & 18.88 \\
& & 170 & & & \\
\hline
\end{tabular}

$$
\mathrm{R}=0.12063 \times 10^{6} \quad \rho=4.1210 \times 10^{7} \quad \mathrm{~J}=9.628
$$

Coefficient of electrical conductivity $(\sigma)=2.426 \times 10^{-8}$ mho.m-1

Table 15. Electrets properties of renal stone at $65^{\circ} \mathrm{C}, \mathrm{A}=9 \times 10^{-6} \mathrm{~m}^{2}, \mathrm{~L}=3 \times 10^{-3} \mathrm{~m}$

\begin{tabular}{|c|c|c|c|c|c|}
\hline S.NO & $\begin{array}{l}\text { Voltage applied } \\
\text { in Volts (V). }\end{array}$ & $\begin{array}{l}\text { Current measured in micro } \\
\text {-amperes (I). }\end{array}$ & $\begin{array}{c}\text { Resistance } \\
10^{+6} \\
(\mathrm{Ohm})\end{array}$ & $\begin{array}{l}\text { Resistivity in } 10^{7} \\
\text { Ohm. M }(\rho) .\end{array}$ & $\begin{array}{l}\text { Current } \\
\text { density } \\
(\mathrm{J}) \text {. } \\
\text { Amp } / \mathrm{m}^{2}\end{array}$ \\
\hline 1. & 2 & 15 & 0.1338 & 3.7933 & 1.66 \\
\hline 2. & 4 & 25 & 0.16 & 5.333 & 2.77 \\
\hline 3. & 6 & 30 & 0.2 & 6.667 & 3.33 \\
\hline 4. & 8 & 38 & 0.2105 & 7.0167 & 4.22 \\
\hline 5. & 10 & 50 & 0.2 & 6.6667 & 5.55 \\
\hline 6. & 12 & 57 & 0.2105 & 7.0167 & 6.33 \\
\hline 7. & 14 & 65 & 0.2154 & 7.1800 & 7.22 \\
\hline 8. & 16 & 74 & 0.2162 & 7.2666 & 8.22 \\
\hline 9. & 18 & 82 & 0.2195 & 7.3167 & 9.11 \\
\hline 10. & 20 & 100 & 0.2 & 6.6667 & 11.11 \\
\hline
\end{tabular}

$$
\mathrm{R}=0.19654 \mathrm{X} 10^{6} \quad \rho=6.4863 \times 10^{7} \quad \mathrm{~J}=5.952
$$

Coefficient of electrical conductivity $(\sigma)=1.542 \times 10^{-8}$ mho.m-1 
Table 16. Electrets properties of renal stone at $99^{\circ} \mathrm{C}, \mathrm{A}=9 \times 10^{-6} \mathrm{~m}^{2}, \mathrm{~L}=3 \times 10^{-3} \mathrm{~m}$

\begin{tabular}{|c|c|c|c|c|c|}
\hline S.NO & $\begin{array}{l}\text { Voltage applied } \\
\text { in Volts }(\mathrm{V}) .\end{array}$ & $\begin{array}{l}\text { Current measured in } \\
\text { micro -amperes (I). }\end{array}$ & $\begin{array}{c}\text { Resistance } \\
10^{+6} \\
(\mathrm{Ohm})\end{array}$ & $\begin{array}{l}\text { Resistivity in } 10^{7} \\
\text { Ohm. M }(\rho) .\end{array}$ & $\begin{array}{l}\text { Current } \\
\text { density }(J) \text {. } \\
\text { Amp/m } \text { m }^{2}\end{array}$ \\
\hline $\begin{array}{c}1 . \\
2 . \\
3 . \\
4 . \\
5 . \\
6 . \\
7 . \\
8 . \\
9 . \\
10 . \\
11 . \\
12 . \\
13 . \\
14 .\end{array}$ & $\begin{array}{l}2 \\
4 \\
5 \\
6 \\
7 \\
8 \\
9 \\
10 \\
11 \\
12 \\
13 \\
14 \\
15 \\
16\end{array}$ & $\begin{array}{c}8 \\
15 \\
20 \\
26 \\
34 \\
38 \\
42 \\
45 \\
50 \\
58 \\
65 \\
70 \\
74 \\
78\end{array}$ & \begin{tabular}{|l}
0.25 \\
0.2667 \\
0.25 \\
0.2308 \\
0.2059 \\
0.2105 \\
0.2145 \\
0.2222 \\
0.22 \\
0.2069 \\
0.2350 \\
0.2 \\
0.2027 \\
0.2
\end{tabular} & $\begin{array}{c}8.333 \\
8.8900 \\
8.3333 \\
7.6933 \\
6.8633 \\
7.0167 \\
7.1433 \\
7.4000 \\
7.4000 \\
6.8967 \\
7.8333 \\
6.6667 \\
6.7567 \\
6.6667\end{array}$ & $\begin{array}{l}0.88 \\
1.66 \\
2.22 \\
2.88 \\
3.77 \\
4.22 \\
4.66 \\
5.00 \\
5.55 \\
6.44 \\
7.22 \\
7.77 \\
8.22 \\
8.66\end{array}$ \\
\hline
\end{tabular}

Coefficient of electrical conductivity $(\sigma)=1.3475 \times 10^{-8} \quad$ mho.m-1

\section{Results and Discussion}

We are unable to measure the hall coefficient of the renal stones. The stones containing collagen like micro-protein and apatite also have some organic matrix like protein at 5\% of the total weight [13]. The protein matrix is clearly visible under a scanning microscope. The D.C electrical conductivity of the renal stones are compared and calculated with the standard ionic conductors [11] and semiconductors [12].

The ionic conduction for the renal stone is

$$
\sigma=\sigma_{1} \exp ^{(\mathrm{A} / \mathrm{KT})}+\sigma_{2} \exp ^{(-\mathrm{B} / \mathrm{KT})}
$$

Where $\sigma_{1}, \sigma_{2}$ are the zero field conductivity, A and B are constants. The two exponential terms are nature of normal conductors. 
In semiconductors the conductivity

$\sigma=\sigma_{0} \exp ^{(-\mathrm{Eg} / 2 \mathrm{KT})}$

Here $\sigma_{0}$ is the zero field conductivity and Eg is the activation energy of the conductors at a particular temperature (T). Because of practical inconvenience of electron microscope (SEM), a fine powder of kidney stone was observed under a high resolution optical microscope and shows fibrils of protein. Thus renal stones can be regarded as a mixture of semi conducting materials like $\mathrm{N}$ type and $\mathrm{P}$ type, but totally it behaves like $\mathrm{N}$ type material or conductors. Hence, conductivity of a kidney stone shows it may be interpreted in terms of a partially compensated semiconductor.

Available mechanism for conduction of renal stones may be sought with the help of various scattering mechanism of conductors and semiconductors [14]. The conductivity of samples depends upon the scattering by lattice vibrations. In conductor the curve between conductivity and temperature should be straight line, but in semiconductor, it is not in usual [15].

The TSP, TSD data of kidney stones also give added information about its conduction mechanism $[2,3]$. For the TSP, TSD conductivity of a sample, the changing current is composed of three components, which is conduction, polarization and depolarization. When temperature increases, the conductivity of a samples is increased, the polarization and depolarization peaks merge in the conduction current or only a part of it is observable [16]. The voltage dependence of conductivity decreases with rise of temperature $(T)$. The current density decreases, which shows the conduction is in non-ohmic. This change suggests a warm electron effect [17]. At higher temperature, the current density is directly proportional to voltage and gives the ohmic behaviors. This is clear that for a sample at higher temperature, the thermal energy difference between the charge carriers and lattices are relatively low or due to asymmetric effect [18] formed in inside the crystals of calcium oxalates and calcium phosphates. 
Table 17. Electrical parameters of renal stone samples

\begin{tabular}{|c|c|c|c|c|c|}
\hline S No & Stones & $\begin{array}{l}\text { Temperature } \\
\text { in degree } \\
\text { Celsius }\end{array}$ & $\mathrm{R}$ in ohms & $\begin{array}{l}\rho \\
\text { Ohm-m }\end{array}$ & $\begin{array}{l}\mathrm{J} \\
\mathrm{Amp} / \mathrm{m}^{2}\end{array}$ \\
\hline \multirow[t]{3}{*}{1} & \multirow[t]{3}{*}{ A } & 30 & $1.149 \times 10^{6}$ & $7.5591 \times 10^{7}$ & 0.0409 \\
\hline & & 60 & $1.4207 \times 10^{6}$ & $9.3469 \times 10^{7}$ & 0.033 \\
\hline & & 96 & $1.149 \times 10^{6}$ & $15.2715 \times 10^{7}$ & 0.0202 \\
\hline \multirow[t]{3}{*}{2} & \multirow[t]{3}{*}{ B } & 28 & $1.149 \times 10^{6}$ & $8.02725 \times 10^{7}$ & 4.2525 \\
\hline & & 65 & $0.05702 \times 10^{6}$ & $10.3676 \times 10^{6}$ & 3.07 \\
\hline & & 98 & $0.0885 \times 10^{6}$ & $1.6097 \times 10^{6}$ & 2.087 \\
\hline \multirow[t]{3}{*}{3} & \multirow[t]{3}{*}{$\mathrm{C}$} & 30 & $0.6486 \times 10^{6}$ & $14.4151 \times 10^{6}$ & 0.6569 \\
\hline & & 58 & $1.1826 \times 10^{6}$ & $2.5938 \times 10^{6}$ & 0.484 \\
\hline & & 95 & $1.7198 \times 10^{6}$ & $3.9577 \times 10^{6}$ & 0.464 \\
\hline \multirow[t]{3}{*}{4} & \multirow[t]{3}{*}{$\mathrm{D}$} & 28 & $0.2743 \times 10^{6}$ & $5.4874 \times 10^{7}$ & 2.049 \\
\hline & & 70 & $0.3517 \times 10^{6}$ & $7.0342 \times 10^{7}$ & 1.885 \\
\hline & & 98 & $0.4864 \times 10^{6}$ & $0.9728 \times 10^{8}$ & 1.246 \\
\hline \multirow[t]{3}{*}{5} & \multirow[t]{3}{*}{$\mathrm{E}$} & 28 & $0.12063 \times 10^{6}$ & $4.1210 \times 10^{7}$ & 9.628 \\
\hline & & 65 & $0.19654 \times 10^{6}$ & $6.4863 \times 10^{7}$ & 5.952 \\
\hline & & 99 & $0.22 \times 10^{6}$ & $7.4209 \times 10^{7}$ & 4.399 \\
\hline
\end{tabular}


Table 18. Thermal and Electrical conductivity relation with the Temperature. Loraznts constant

$\left(\mathrm{L}_{\mathrm{o}}\right)=2.44 \mathrm{x} 10^{-8} \mathrm{w} \Omega \mathrm{k}^{-2}$

\begin{tabular}{|c|c|c|c|c|}
\hline SNo & $\begin{array}{l}\text { Name of the } \\
\text { sample }\end{array}$ & $\begin{array}{l}\text { Conductivity } \\
(\sigma) \mathrm{X}^{-8} 0^{-8} \\
\mathrm{mho}^{-\mathrm{m}^{-1}}\end{array}$ & $\begin{array}{l}\text { Temperature } \\
\text { in degree Celsius }\end{array}$ & $\begin{array}{l}\text { Thermal } \\
\text { conductivity } \\
\mathrm{K}=\sigma \mathrm{L}_{\mathrm{O}} \mathrm{T} \quad \mathrm{X} 10^{-14} \\
\mathrm{~W} / \mathrm{M} / \mathrm{K}\end{array}$ \\
\hline \multirow[t]{3}{*}{1} & \multirow[t]{3}{*}{ A } & 1.328 & 30 & 0.9721 \\
\hline & & 1.0698 & 60 & 1.5662 \\
\hline & & 0.6548 & 96 & 15.3381 \\
\hline \multirow[t]{3}{*}{2} & \multirow[t]{3}{*}{ B } & 12.457 & 28 & 8.5106 \\
\hline & & 9.645 & 65 & 15.297 \\
\hline & & 6.212 & 98 & 14.551 \\
\hline \multirow[t]{3}{*}{3} & \multirow[t]{3}{*}{ C } & 0.6937 & 30 & 0.5077 \\
\hline & & 0.3855 & 58 & 0.5456 \\
\hline & & 0.2661 & 95 & 6.1682 \\
\hline \multirow[t]{3}{*}{4} & \multirow[t]{3}{*}{ D } & 1.822 & 28 & 1.2448 \\
\hline & & 1.422 & 70 & 2.4288 \\
\hline & & 0.10279 & 98 & 2.4941 \\
\hline \multirow[t]{3}{*}{5} & \multirow[t]{3}{*}{ E } & 2.426 & 28 & 1.6570 \\
\hline & & 1.542 & 65 & 2.4456 \\
\hline & & 1.3475 & 99 & 3.255 \\
\hline
\end{tabular}

Table 19. Temperature coefficient of the renal stone at different temperatures.

\begin{tabular}{|l|l|l|l|}
\hline Sample & $\begin{array}{l}\text { Temperature } \\
\text { minimum in } \\
\text { degree Celsius }\end{array}$ & $\begin{array}{l}\text { Temperature } \\
\text { Maximum in } \\
\text { degree Celsius }\end{array}$ & $\begin{array}{l}\text { Temperature coefficient } \\
\text { of the sample. } \\
\alpha=\left(\mathrm{R}_{2}-\mathrm{R}_{1}\right) /\left(\mathrm{R}_{1} \mathrm{~T}_{2}-\mathrm{R}_{2} \mathrm{~T}_{1}\right)\end{array}$ \\
\hline \multirow{2}{*}{$\mathrm{A}$} & 30 & 60 & 0.01032 \\
\cline { 2 - 4 } & 30 & 96 & 0.02882 \\
\hline \multirow{3}{*}{ B } & 28 & 65 & 0.01010 \\
\cline { 2 - 4 } & 28 & 98 & 0.0239 \\
\hline C & 30 & 95 & 0.09122 \\
\cline { 2 - 4 } & 58 & 95 & 0.04266 \\
\hline D & 28 & 98 & 0.0159 \\
\cline { 2 - 4 } & 28 & 70 & 0.00827 \\
\hline E & 28 & 99 & 0.01718 \\
\cline { 2 - 4 } & 28 & 65 & 0.03642 \\
\hline
\end{tabular}




\section{Conclusion}

All the renal stones thermal conductivity, electrical conductivity and temperature co-efficient are measured. At higher temperature, the thermal conductivity of all the samples is constant, but the electrical conductivity varied. All the stones are positive temperature coefficient materials. The entire samples are high conductivity at low temperature, but when the temperature increases, the conductivity decreases, except E stone.

\section{References}

[1].Simionescu.C. etal. Topics in biochemistry and Biogenetics, John Willy and sons, New York, 27,P 151-204 (1976).

[2]. Yagik.Ya. Etal, Electrets studies kidney stones, in proc. Solid State Phys Sym. BARC, Bombay, India, P 23-25,27c.,205 (1984)

[3].Yagik.Ya etal. Bioelectrets of renal calculi (kidney stone) in proc. $5^{\text {th }}$ Int. Sym. Electrets Heidelberg, ,819-824.(1985)

[4].Yagik.Ya.etal. The electrets affect kidney stones, Biomaterials, 8(b), P 503-505.(1987)

[5]. Vermuclen.C.W. etal Experimental observation on the pathogeneses of urinary calculi.J.urology,,95,681.(1964)

[6].Lowell.J. Surface conduction and absorption currents in polymers. J. Phys. D, 15,109.(1982)

[7].Talwar.I.M. etal. Electrical conductivity studies in polyvinyl chloride.Ind.J.Pure.Appl.Phys. 7,681 (1982).

[8].Sharma.B.JetalElectrical conduction in kaptron polymate film at higher electrical field, Polymer, 73,17 (1987).

[9].Consh .J. etal Ionic conductivity of silver chloride single crystals, J.Phys.Chem.solids, 33,1799 (1972).

[10].Ramasastry.C. etal, Electrical conductivity in sodium chloride crystals Proc.R.Soc.A 325,34715.(1971)

[11].Vanderzerd.A.Solid state physical Electronics printer's hall of India, New Delhi, 492 (1971).

[12]. Pething.R. Dielectrics \& electronic properties of biological materials. John Willy and sons, New York P 303.(1979)

[13]. Jethi.R.K. etal. Effect of aqueous electrets of an aurvedic compounds properties on minerlization and demineralization reaction. Ind. J medical research, 78, P 422-25.(1983)

[14]. Kareev.P.S. Semiconductor physics Mir publishers, Moscow, P 428-436. (1974).

[15]. Greeve.P.S. Physics and technology of semiconductor devices. . John Willy and sons, Newyork, P109 (1967).

[16] Mckeever,S.W.S. etal. Thermally stimulated currents in dielectric. J.phy. D, 8, P 1520. (1975)

[17]. Black more. J.S. Solid state physics $2^{\text {nd }}$ edi. W.B. Sawnders Company Tokyo, Japan, P-357358.(1974)

[18]. Glasstone .S. An introduction to electro chemistry D.Van No strand, P 878-114. (1965) 